\title{
DIMENSION FUNCTIONS FOR FRACTAL SETS ASSOCIATED TO SERIES
}

\author{
MANUEL MORÁN
}

(Communicated by Andrew Bruckner)

\begin{abstract}
In this article we analyze suitable dimension functions for the measure of fractal sets associated to certain absolutely convergent series of vectors of $\mathbb{R}^{n}$. The complex binomial series provides an example of a family of fractals, all of them with the same Hausdorff dimension but with a totally ordered family of suitable dimension functions indexed by a parameter with range in a real interval. We also show a construction, based on sets associated to series, to obtain fractal sets with given dimension function.
\end{abstract}

\section{INTRODUCTION}

We start by recalling that the set $\mathscr{H}$ of dimension functions is the set of functions $f: \mathbb{R}^{+} \cup\{0\} \rightarrow \mathbb{R}^{+} \cup\{0, \infty\}$, monotonic increasing and continuous on the right. There is a Hausdorff measure $H^{f}$ for each $f \in \mathscr{H}$ which is defined in the following way: for every $A \subseteq \mathbb{R}^{n}$ and $\delta>0$ we define

$$
H_{\delta}^{f}(A)=\inf \left\{\sum_{i \in \mathbb{N}} f\left(\left|U_{i}\right|\right):\left\{U_{i}\right\} \text { is a countable } \delta \text {-cover of } A\right\}
$$

(we use $|U|$ for diameter of $U$ ). Then

$$
H^{f}(A)=\lim _{\delta \rightarrow 0} H^{f}(A)
$$

A partial ordering can be defined in $\mathscr{H}$ by

$$
f \prec g \Leftrightarrow \lim _{h \rightarrow 0} g(t) / f(t)=0 .
$$

If $f \prec g$ then $H^{f}(C)<\infty$ implies $H^{g}(C)=0$ and $H^{g}(C)>0$ implies $H^{f}(C)=\infty$.

For $f(x)=x^{s}, s \geq 0, H^{f}$ is the usual $H^{s}$ measure. One identifies the dimension function $x^{s}$ with its exponent $s$, so that $x^{s} \prec x^{t}$ is equivalent to $s<t$. For a detailed account of dimension functions and their associated measures see [1].

Received by the editors June 9, 1992.

1991 Mathematics Subject Classification. Primary 28A78, 28A80.

Key words and phrases. Dimension, fractal. 
Let $\sum_{i=1}^{\infty} a_{i}$ be an absolutely convergent series of vectors of $\mathbb{R}^{n}$. The set

$$
C=\left\{\sum_{i \in I} a_{i}: I \subseteq \mathbb{N}\right\}
$$

is the set associated to $\sum_{i=1}^{\infty} a_{i}$. Under certain conditions on the series, $C$ is a fractal set. Some of its properties (among them its Hausdorff dimension) have been analyzed in [2]. Sets of this kind appear as the support of the limit distributions of infinite convolutions of discrete measures (see [3-5])-there being open problems about the absolute continuity of the limit distribution.

The set $C$ can be decomposed, for every $k \in \mathbb{N}$, as

$$
C=\bigcup_{x \in C_{k}^{-}}\left\{x+C_{k}^{+}\right\}
$$

where

$$
C_{k}^{-}=\left\{\sum_{i \in I} a_{i}: I \subseteq\{0,1,2, \ldots, k\}\right\}
$$

and

$$
C_{k}^{+}=\left\{\sum_{i \in I} a_{i}: I \subseteq\{k+1, k+2, \ldots\}\right\} .
$$

Two basic parameters in this decomposition are $\rho_{k}=$ Card $C_{k}^{-}$, which gives the number of translated copies of $C_{k}^{+}$by vectors of $C_{k}^{-}$which made up $C$, and $R_{k}=\sum_{i>k}\left|a_{i}\right|$, which gives an upper bound for $\left|C_{k}^{+}\right| / 2$ (here $\left|a_{i}\right|$ denotes the modulus of $a_{i}$ ).

\section{DIMENSION FUNCTION FOR FRACTAL SETS ASSOCIATED TO SERIES}

The scaling between the diameters $\left|C_{k}^{+}\right|$and $\left|C_{k+1}^{+}\right|$is not independent of $k$ (except in the case in which $a_{i}=c r^{i}$ with $r \in \mathbb{C}$ or $r \in \mathbb{R}$ ). For this reason sets associated to series provide a simple example of an essentially nonselfsimilar deterministic family of fractals. The dimension functions $x^{s}$ do not satisfactorily measure the sets associated to series. Our aim in this section is to show how we can obtain, in some cases, other dimension functions more appropriated.

Theorem. Let $\sum a_{i}$ be an absolutely convergent series in $\mathbb{R}^{n}, C$ its associated set, and $f \in \mathscr{H}$. Then:

(i) $\operatorname{Liminf} f\left(2 R_{i}\right) \rho_{i}=s \Rightarrow H^{f}(C) \leq s$.

(ii) Liminf $f\left(R_{i}\right) \rho_{i}=s>0$ and $\left|a_{i}\right| \geq R_{i}$ for all $i$ large enough $\Rightarrow$ $H^{f}(C)>0$.

Proof. (i) Fix $\delta, \varepsilon>0$ and take $k$ such that $\left|R_{k}\right|<\delta$ and $f\left(2 R_{k}\right) \rho_{k}<s+\varepsilon$. Then $\bigcup_{x \in C_{k}^{-}}\left\{x+C_{k}^{+}\right\}$is a $\delta$-covering of $C$. Hence,

$$
H_{\delta}^{f}(C) \leq \sum_{x \in C_{k}^{-}} f\left(\left|x+C_{k}^{+}\right|\right) \leq f\left(2 R_{k}\right) \rho_{k}<s+\varepsilon .
$$

Letting $\varepsilon$ and $\delta$ go to zero we get $H^{f}(C) \leq s$. 
(ii) This proof is an adaptation of [2, Proof of Theorem 3]. The steps for both proofs are the same, but here we must deal with the problems derived from the overlapping, compatible with our hypothesis $\left|a_{i}\right| \geq R_{i}$ (in [2] such possibility was denied by the assumption $\left.\lim \sup R_{i} /\left|a_{i}\right|<1\right)$.

As in the quoted reference, the first step is to prove that, for any ball $B$ of radius $\rho$, we can select a $p \in \mathbb{N}$ such that $R_{p}<\rho$ and the maximum number $q$ of sets $x+C_{p}^{+}$, with $x \in C_{p}^{-}$, intersected by $B$, is independent of $\rho$. Let

$$
r=\min \left\{|x-y| / 2: x, y \in C_{p}^{-}, x \neq y\right\} .
$$

It is proved in [2] that if $r \geq \rho$ then $q<3^{n}$ and if $r<\rho$ then

$$
q \leq\left[3\left(\left|a_{p}\right|+R_{p}\right) / r\right]^{n} \text {. }
$$

By using this inequality it is also proved in [2] that if we fix a positive number $M<1$ and $R_{p} /\left|a_{p}\right|<M$ then

$$
q \leq[12 /(1-M))^{n} \text {. }
$$

Assume now that $R_{p} /\left|a_{p}\right| \geq M$. If $x=a_{p}$ and $y=0$ then $|x-y|=\left|a_{p}\right|$. For the remaining cases in which $x, y \in C_{p}^{-}$and $x \neq y$

$$
\begin{aligned}
|x-y| & \geq \min \left\{\left|a_{i}\right|-\sum_{k=i+1}^{p}\left|a_{k}\right|: 0 \leq i \leq p\right\} \\
& =\min \left\{\left|a_{i}\right|-R_{i}+R_{p}: 0 \leq i \leq p\right\} \geq R_{p}
\end{aligned}
$$

so that $r \geq R_{p} / 2$. Hence,

$$
q \leq 6^{n}\left(\left|a_{p}\right| / R_{p}+1\right)^{n} \leq 6^{n}(1 / M+1)^{n},
$$

and $q$ is always bounded independently of $\rho$.

The next step is to prove that, if $C$ is not a finite set, there is a Borel probability distribution $\mu$ supported by $C$ such that $\mu\left(x+C_{i}^{+}\right)=1 / 2^{i+1}$ for all $i \in \mathbb{N}$ and $x \in C_{i}^{-}$. We claim that if there is some overlapping it must occur over a countable set $\mathscr{O}$. To prove our claim assume $x=\sum_{i \in I} a_{i}=\sum_{i \in J} a_{i}$ with $I \neq J$. We can also assume $a_{i} \neq 0$ for all $i \in \mathbb{N}$. Let $k$ be the least number in $D=(I-J) \cup(J-J)$. We can assume $i \in I$. Then $a_{k}=\sum_{i=k+1}^{\infty} \delta_{i} a_{i}$, where $\delta_{i}=-1$ when $i \in(I-J), \delta_{i}=0$ when $i \in \mathbb{N}-D$, and $\delta_{i}=1$ when $i \in(J-I)$. Hence,

$$
\left|\sum_{i=k+1}^{\infty} \delta_{i} a_{i}\right| \geq R_{k},
$$

and this requires all $\delta_{i} a_{i}, i \in D$, to be collinear and $D=\{k, k+1, k+2, \ldots\}$, so that we can assume the series to be a real series except for a finite set of terms. We can assume that $a_{k}$ is positive. Then, by virtue of the above inequality, $a_{i}<0$ for all $i \geq k, i \in I$ and $a_{i}>0$ for all $i \geq k, i \in J$. This shows that the set $I$ is uniquely determined except for the finite set of indices $\{i \in \mathbb{N}: i<k\}$. This proves that $\mathscr{O}$ is countable, since it can be obtained as a countable union of finite sets. Then we can distribute a unit mass uniformly over the family of sets $\left\{x+C_{i}^{+}-\mathscr{O}\right\}, i \in \mathbb{N}, x \in C_{i}^{-}$, by defining $\mu\left(\left\{x+C_{i}^{+}\right\}-\mathscr{O}\right)=2^{-i-1}$, and this distribution can be extended to the Borel sets as required (see [6, Chapter 1, Proposition 1.7]). 
We can now prove assertion (ii). If $C$ is a finite set then $R_{i}=0$ for all $i$ large enough and $f(0)$ must be positive. Therefore, this case is trivial. If $C$ is not finite then, by using in a standard way (see [2]) the two previous results proved above, it can be shown that, for any ball $B$ with radius small enough, $\mu(B)<c f(|B|)$, where $c$ is a positive constant independent of $B$. We then get $H^{f}(C)>0$ as a straightforward consequence of the "distribution mass principle" (see [6]).

The following corollary of the theorem above states conditions for a function $f$ to be an adequate function dimension for the set $C$ associated to the series $\sum a_{i}$.

Corollary. Let $\sum a_{i}$ be a series of vectors of $\mathbb{R}^{n}$ with $\left|a_{i}\right| \geq R_{i}$ for $i$ large enough. Let $f \in \mathscr{H}$ be such that $0<\liminf f\left(\left|a_{i}\right|\right) 2^{i}=s<\infty$. Then $0<$ $H^{f}(C)<\infty$. Moreover, if $\left|a_{i}\right| \geq R_{i}$ for all $i$ then $H^{f}(C) \leq 4 s$.

Proof. This result is a direct consequence of the theorem, taking into account that if $\left|a_{i}\right| \geq R_{i}$ for all $i$ large enough then $\rho_{i}=K 2^{i}$, where $K$ is a positive constant.

With this corollary we can construct a dimension function giving finite and positive measure to the set $C$ associated to any series $\sum a_{i}$ such that $\left|a_{i}\right| \geq R_{i}$ for $i$ large enough. If we assume this last condition, the functional equation

$$
f\left(\left|a_{i}\right|\right)=2^{-i}, \quad f \in \mathscr{H},
$$

for all $i$ large enough, solves the problem of finding a dimension function giving finite and positive measure to $C$. Such an equation is solved by the function

$$
\begin{cases}f(x)=1 / 2^{i} & \text { when }\left|a_{i}\right| \leq x<\left|a_{i-1}\right|, i \geq 1 \\ f(x)=1 & \text { when } x \geq\left|a_{0}\right| \\ f(0)=0 & \end{cases}
$$

\section{EXAMPLES AND APPLICATIONS}

(a) In some cases the above functional equation can be solved explicitly in terms of simple analytic functions. Let $\sum\left(\begin{array}{l}\alpha \\ i\end{array}\right) z^{i}$ be a binomial complex series with $\alpha \leq-1$ and $0<|z| \leq 1-2^{1 / \alpha}$. Let $C$ be the set associated to this series. For $s=-\log 2 / \log |z|$ let $f \in \mathscr{H}$ be given by

$$
f(x)=(-\log x)^{(\alpha+1) s} x^{s}
$$

for $x \geq 0$ and $f(0)=0$. Then the following proposition holds.

Proposition 1. $\operatorname{Dim}(C)=s$ and $0<H^{f}(C)<\infty$.

Proof. First we prove that $R_{i} /\left|\left(\begin{array}{c}\alpha \\ i\end{array}\right) z^{i}\right| \leq 1$.

$$
\begin{aligned}
R_{i}||\left(\begin{array}{c}
\alpha \\
i
\end{array}\right) z^{i} \mid & =\frac{\sum_{k>i}\left|\left(\begin{array}{c}
\alpha \\
i
\end{array}\right) z^{k}\right|}{\left.\mid \begin{array}{c}
\alpha \\
i
\end{array}\right) z^{i} \mid}=\sum_{k>i}\left|\frac{(\alpha-i)(\alpha-i-1) \cdots(\alpha-k+1)}{k(k-1) \cdots(i+2)(i+1)} z^{k-i}\right| \\
& =\sum_{k>i} \frac{(-\alpha+i)(-\alpha+i+1) \cdots(-\alpha+k-1)}{(i+1)(i+2) \cdots k}|z|^{k-i} \\
& \leq \sum_{k>i} \frac{(-\alpha)(-\alpha+1) \cdots(-\alpha+k-i-1)}{1 \cdot 2 \cdots(k-i)}|z|^{k-i}
\end{aligned}
$$


where the last inequality holds because if $\alpha \leq-1$ then for $i, p \in \mathbb{N}$

$$
(-\alpha+i+p) /(i+1+p) \leq(-\alpha+p) /(1+p) .
$$

We can use the fact that

$$
\sum_{k>i} \frac{(-\alpha)(-\alpha+1) \cdots(-\alpha+k-i-1)}{1 \cdot 2 \cdots(k-i)}|z|^{k-i}=(1-|z|)^{\alpha}-1
$$

and, since $|z| \leq 1-2^{1 / \alpha}$, we get $(1-|z|)^{\alpha}-1 \leq 1$ as required.

We now proceed to prove

$$
K_{1}<\log \left|\left(\begin{array}{c}
\alpha \\
i
\end{array}\right)\right|+(\alpha+1) \log i<K_{2}
$$

with $K_{1}, K_{2}$ constants. We will use this fact later.

$$
\begin{aligned}
\log \left|\left(\begin{array}{c}
\alpha \\
i
\end{array}\right)\right| & =\log \frac{(-\alpha)(-\alpha+1) \cdots(-\alpha+i-1)}{1 \cdot 2 \cdots i}=\sum_{k=1}^{i} \log \left(1+\frac{(-\alpha-1)}{k}\right) \\
& =\sum_{k=1}^{i}\left[\frac{-\alpha-1}{k}-\int_{0}^{(-\alpha-1) / k} \frac{t}{1+t} d t\right] .
\end{aligned}
$$

Since

$$
\int_{0}^{(-\alpha-1) / k} \frac{t}{1+t} d t \leq \int_{0}^{(-\alpha-1) / k} t d t=\frac{(-\alpha-1)^{2}}{2 k^{2}}
$$

we can write

$$
\sum_{k=1}^{i} \frac{-\alpha-1}{k}-\sum_{k=1}^{i} \frac{(-\alpha-1)^{2}}{2 k^{2}} \leq \log \left|\left(\begin{array}{c}
\alpha \\
i
\end{array}\right)\right| \leq \sum_{k=1}^{i} \frac{-\alpha-1}{k} .
$$

Then, since $\lim \sum_{k=1}^{i}[(\alpha-1) / k+(\alpha+1) \log i]=$ const and $\sum_{k=1}^{i}(-\alpha-1)^{2} / 2 k^{2}$ is convergent, we get the required result.

The inequalities $0<\lim \inf f\left(\left|\left(\begin{array}{c}\alpha \\ i\end{array}\right) z^{i}\right|\right) 2^{i}<\infty$ can be shown to be true by direct computation, using the fact that the sequence $\log a_{i}+(\alpha+1) \log i$ is bounded. Lastly, for any $t<s, x^{t} \prec f(x)$ while, for any $s<t, f(x) \prec x^{t}$. This shows that the Hausdorff dimension of $C$ is $s$. Q.E.D.

We remark that if $\alpha<-1$ then $x^{s} \prec f(x)$. Therefore, $0<H^{s}(C)<\infty$ only when $\alpha=-1$ and, for every $\alpha<-1, H^{s}(C)=\infty$. Moreover, if we write

$$
f_{\alpha}=(-\log x)^{(\alpha+1) s} x^{s}
$$

then, for $\alpha<\alpha^{\prime} \leq-1, f_{\alpha} \succ f_{\alpha^{\prime}}$. Thus, if $C_{\alpha, z}$ is the fractal set associated to the series $\sum\left(\begin{array}{l}\alpha \\ i\end{array}\right) z^{i}$ and $S_{r}=\{z \in \mathbb{C}:|z|=r\}$ with $r<1 / 2$, then all sets in the family

$$
\left\{C_{\alpha, z}\right\}, \quad z \in S_{r}, \log 2 / \log (1-|z|) \leq \alpha \leq-1,
$$

have the same Hausdorff dimension, but the functions giving them finite and positive measure constitute a continuum power family of totally ordered dimension functions.

(b) In the case of the exponential complex series $\sum z^{i} / i$ ! the functional equation $f\left(\left|a_{i}\right|\right)=2^{-i}$ can be solved in the following way: Let $g$ be a real function defined over $\mathbb{R}^{+}$by $g(t)=|z|^{t} / \Gamma(t)$, where $\Gamma(t)$ is the Gamma function. Then there is an $i_{0} \in \mathbb{N}$ such that $g$ is analytic and strictly decreasing over $\left(i_{0}, \infty\right)$. 
Let $f(x)=2^{-g^{-1}(x)}$. Then $g^{-1}\left(|z|^{i} / i !\right)=i$ for all $i \in \mathbb{N}$ such that $i>i_{0}$. Hence, $f\left(\left|a_{i}\right|\right)=2^{-i}$ for such $i$ and, if we define $f(0)=0, f$ is a dimension function analytic in $\left(0,\left|a_{i_{0}}\right|\right)$ and giving finite and positive measure to the fractal set associated to $\sum z^{i} / i$ ! .

(c) The corollary above poses the inverse problem of finding what functions can give finite and positive measure to the set associated to some series. The following proposition gives sufficient conditions for such functions.

Proposition 2. (a) Let $f \in \mathscr{H}$. For all $i \in \mathbb{N}$ let

$$
x_{i}=\inf \left\{x: f(x) \geq 2^{-i}\right\} \text {. }
$$

If either $x_{i}=0$ for some $i$ or $2 \leq x_{i} / x_{i+1}$ for all $i$ large enough, then there exists an absolutely convergent real series $\sum a_{i}$ with associated set $C$ such that $0<H^{f}(C)<\infty$.

(b) Let $f \in \mathscr{H}$ be concave and nonidentically zero in some interval $(0, b)$. Then $f$ verifies the hypotheses of (a).

Proof. (a) If $x_{i}=0$ for some $i$ then any finite series trivially satisfies the required conditions. Assume now that there exists an $i_{0}$ such that $2 \leq x_{j} / x_{j+1}$ for all $j \geq i_{0}$. Therefore, $2^{i} \leq x_{i_{0}} / x_{i_{0}+i}$ so that $x_{j} 2^{j} \leq x_{i_{0}} 2^{i_{0}}$ for all $j \geq i_{0}$. We obtain the required series $\sum a_{i}$ by taking $x_{i}-x_{i+1}$ as $a_{i}$. Then $R_{i}=$ $x_{i+1}-\lim x_{i}=x_{i+1}$, since $\left\{x_{i}\right\}$ is decreasing and $2^{i} x_{i}$ is bounded. For all $i>i_{0}, x_{i} \neq x_{i-1}$. Then, by virtue of the continuity of $f$ on the right and the definition of $x_{i}$, we have $2^{-i} \leq f\left(x_{i}\right)<2^{-i+1}$. Hence,

$$
\liminf f\left(R_{i}\right) 2^{i}=\liminf f\left(x_{i+1}\right) 2^{i} \geq 2^{-1}
$$

and

$$
\liminf f\left(2 R_{i}\right) 2^{i} \leq \liminf f\left(2 x_{i+1}\right) 2^{i} \leq \liminf f\left(x_{i}\right) 2^{i}<2 .
$$

We also have for all $i>i_{0}$

$$
R_{i} /\left|a_{i}\right|=x_{i+1} /\left(x_{i}-x_{i+1}\right)=\left[x_{i} / x_{i+1}-1\right]^{-1} \leq 1 \text {. }
$$

This completes the proof of (a).

(b) If $f(0) \neq 0$ then $x_{i}=0$ for some $i$. If $y=\lim x_{i}>0$ then $f=0$ in the interval $(0, y)$. Therefore, we can assume that $f(0)=0$ and $y=0$. Let $i_{0} \in \mathbb{N}$ be such that $x_{i_{0}}<b$. We can write $x_{i} / x_{i+1}=f^{-1}\left(2^{-i}\right) / f^{-1}\left(2^{-i-1}\right)$. Then the concavity of $f$ implies $x_{i} / x_{i+1} \geq 2$ for all $i>i_{0}$. Q.E.D.

\section{REFERENCES}

1. C. A. Rogers, Hausdorff measures, Cambridge Univ. Press, London and New York, 1970.

2. M. Morán, Fractal series, Mathematika 36 (1989), 334-348.

3. B. Jessen and A. Wintner, Distribution functions and the Riemann zeta function, Trans. Amer. Math. Soc. 38 (1935), 48-88.

4. P. Erdos, On a family of symmetric Bernoulli convolutions, Amer. J. Math. 62 (1940), 180-186.

5. A. M. Garsia, Arithmetic properties of Bernoulli convolutions, Trans. Amer. Math. Soc. 102 (1962), 409-433.

6. K. Falconer, Fractal geometry, Wiley, New York, 1990.

Departamento de Análisis Económico, Universidad Complutense de Madrid, Campus de Somosaguas, 28223 Madrid, Spain

E-mail address: ececo060sis.ucm.es 